\title{
CHAIN OF WASTES: THE MODERATING ROLE OF MAKING-DO
}

\author{
Marcus C. T. Fireman ${ }^{1}$ and Tarcisio A. Saurin ${ }^{2}$
}

\begin{abstract}
The concept of chain of wastes has been used to describe the systemic impacts of some types of wastes. In turn, making-do has been proposed as a leading waste that triggers other wastes that propagate across a chain of wastes. However, there is scarce empirical basis for that proposition, and current models of chain of wastes are not based on a functional perspective, which hinders the traceability of wastes to production activities. This study presents an application of the Functional Resonance Analysis Method (FRAM) for the modelling of a chain of wastes. Based on FRAM, a chain of wastes was modelled for the gutter assembly process of an airport terminal building. Data collection involved the analysis of documents and an interview with the production manager of the construction project. Results indicated that making-do played a moderating role in the chain of wastes. While it compensated for wastes arising from the initial stages of the chain of wastes (i.e. it prevented a contractual fine from being applied) it created new wastes downstream, with implications for the building's use. The provision of slack resources is presented as an alternative for the prevention and mitigation of the impacts of making-do.
\end{abstract}

\section{KEYWORDS}

Chain of Wastes, Making-do, Slack, Functional Resonance Analysis Method.

\section{INTRODUCTION}

Although the construction industry is widely known for having high levels of wastes, most companies in the sector have not yet addressed this problem with the due emphasis (Bølviken and Koskela, 2016). This is partly due to the high complexity of construction projects, which involve a large number of interactions between the factors that contribute to wastes (Formoso et al., 2015). The term "chain of wastes" has been adopted to refer to these interactions (Bølviken and Koskela, 2016).

The concept of chain of wastes is based on Ohno's (1988) proposition that there is a causal relationship between different types of wastes in a production system. According to Onho (1988) the Toyota Production System focused on controlling seven types of wastes, namely: overproduction; defects; transportation; inventory; over-processing; motion; and waiting. Overproduction was regarded by Ohno as the "primary waste" that triggered the others. In the construction industry, Koskela et al. (2013) argue that makingdo plays a similar role, being the primary waste that triggers others

PhD Student, Building Innovation Research Unit ( NORIE), Federal University of Rio Grande do Sul (UFRGS), Porto Alegre, RS, Brazil, marcus@climbgroup.com.br. orcid.org/0000-0001-5843-4715

2 Associate Professor, Industrial Engineering and Transportation Department. Federal University of Rio Grande do Sul (UFRGS), Porto Alegre, RS, Brazil, saurin@ufrgs.br. orcid.org/0000-0003-2929-5888 
According to Koskela (2004), this type of waste occurs when a task starts without meeting all its preconditions or the task is continued even though at least one precondition is unavailable. Empirical studies conducted in construction have identified that wastes of making-do were behind other wastes, such as unfinished works, transport, and rework (Formoso et al., 2011; Fireman et al., 2013; Ibarra et al., 2016).

Formoso et al. (2015) and Bølviken and Koskela (2016) point out that chains of wastes are not linear. This means that small wastes can have disproportionate consequences - i.e. although making-do may have a small local impact, it can have a large impact on the system as a whole. Despite advances in literature regarding the concept of chain of wastes, previous studies are only conceptual and none of them has modelled a real chain of wastes. Therefore, the applicability and utility of the concept of chain of wastes have not been properly explored.

Against this background, the resilience engineering literature offers an approach known as Functional Resonance Analysis Method (FRAM), which allows for modeling interactions between functions in complex systems (Hollnagel, 2012). Functions are the required activities to produce a certain outcome (Hollnagel, 2012). FRAM has been applied mostly for the identification of safety threats and hazards in healthcare, aviation, and other so-called complex socio-technical systems, even though there have been a few studies in construction (e.g. Saurin, 2016).

Although the use of FRAM in production management has been uncommon, it might assist the study of chain of wastes in construction, as these result from interactions between the managerial and productive functions that take place in construction projects. In order to develop a FRAM model, the description of preconditions for starting a function is necessary. In FRAM, a precondition accounts for conditions that must exist before carrying out a function. While a precondition does not start a function, it should be in place before that (Hollnagel, 2012). As such, FRAM seems to be promising for modelling making-do, as a defining characteristic of this waste is the start of a function without fulfilling all preconditions.

This paper discusses, based on FRAM, the role of making-do in a chain of wastes. This investigation is relevant for two reasons: (i) the assumption of making-do as a primary waste lacks empirical grounding; and (ii) the notion of primary waste is elusive as usually there is no single (or primary) root cause for phenomena that matter in complex socio-technical systems, such as wastes and safety (Hollnagel, 2012) - thus, it is reasonable to expect that making-do itself is underlined by other types of wastes.

\section{THE FUNCTIONAL RESONANCE ANALYSIS METHOD (FRAM)}

According to Hollnagel (2013), FRAM can be used for modelling desired or undesired events that result from the combined variability of functions that make up every day work. FRAM describes the subtle variabilities that characterize work-as-done, which tends to be different from work-as-imagined in procedures, policies, and rules (Clay-Williams et al., 2015).

FRAM application involves the steps as follows (Hollnagel, 2012): (i) to define the purpose of FRAM analysis, which can be, for example, an investigation of a past event, a risk assessment of a new system, or an evaluation of design changes; (ii) to identify and describe the functions of the system according to six aspects (input, output, preconditions, resources, time and control); (iii) to describe the variability of the functions, taking into 
account what is expected to happen (or what happened, in the case of a past event) with the output of each function in terms of time (too early, on time, too late, not at all) and precision (precise, acceptable, imprecise); (iv) to aggregate the variability of individual functions, by assessing couplings between functions - couplings occur between the output of a function and any of the other aspects of downstream functions; and (v) to devise practical measures for improving the work system design, if necessary.

\section{FraM PRINCIPLES}

According to Hollnagel (2013), FRAM is based on the four principles described below:

\section{Principle of equivalence (of successes and failures)}

This considers that success and failure have the same causes. However, while success represents the ability of a group, individuals and organizations to anticipate risks before damage occurs, failures represent the inability of the system to effectively adapt to the complexity of the real world.

\section{Principle of approximate adjustments}

Adjustments of performance are always necessary, whether due to finite resources and time, or underspecified plans. However, performance adjustments are not perfect, and therefore variability is part of everyday work in complex systems.

\section{Principle of emergence}

The variability of multiple functions can combine in unexpected ways, leading to nonlinear effects. Thus, both failure and normal performance are emergent, rather than resulting phenomenon, as they cannot be attributed or explained solely based on the functioning or non-functioning of specific components.

\section{Principle of functional resonance}

The combined everyday variability of various functions can sometimes create a functional resonance, thereby producing unexpected results. Functional resonance is the detectable variability (e.g. accidents or wastes) that otherwise remains hidden in everyday work.

\section{RESEARCH METHOD}

\section{RESEARCH STRATEGY}

A case study was carried out to investigate the role of making-do in a chain of wastes, based on FRAM modelling. The unit of analysis for the case study is a chain of wastes generated during the construction phase and which spread to the phase of use of the building project.

The research was divided into three main phases: (i) scoping of the study and selection of the chain of wastes; (ii) FRAM modelling, following the previously mentioned steps proposed by Hollnagel (2012); and (iii) analysis and discussion of results.

\section{Case Study}

The study took place on construction site A, emphasizing services for a metallic structure of an airport terminal building of approximately $29.500 \mathrm{~m}^{2}$. The company responsible for these works, here referred to as Company $X$, is one of the largest steel structure manufacturers in South America, being responsible for the design, production and assembly of the elements that make up the metallic construction system. The study focused on processes of design, production and assembly of the gutters for capturing rain 
water. The production planning and control system of company $\mathrm{X}$ is based on the Last Planner system, including look-ahead planning and short-term commitment planning.

\section{DATA Collection AND ANALYSIS}

The sources of data involved document analysis and the application of a Critical Decision Method (CDM) interview with the production manager of the construction project. This interview was conducted according to the four phases of CDM (Crandal et al, 2006), namely: (i) incident identification; (ii) timeline verification; (iii) deepening; and (iv) "what if" queries. The duration of the interview was 2.5 hours, providing insight into the chain of wastes which had been identified. As for the analysis of documents, it included commitment planning, standardized operating procedures of construction processes and quality management procedures.

Transcripts of interviews and documents were subjected to a content analysis. Predefined data analysis categories were imposed by the researchers, in order to account for the necessary information for developing a FRAM model - i.e. functions and their six aspects, output variability of functions, and possible couplings between functions.

\section{RESULTS}

\section{THE PROCESS OF INSTALLING GUTTERING}

The installation of gutters includes a set of managerial and production activities that involves company $\mathrm{X}$, its supplier and the client. The process starts after the client approves the gutters initial design. Then, the design department of company $\mathrm{X}$ drafts the detailed design of the solution and, when finalized, the company's planning department orders the production of the components. This production is partly carried out by company X (modules of 3-meter gutters) and partly is outsourced (threaded bar and metallic angle brackets). The outsourced components are sent to company X's factory and stored there until the deadline set by the schedule for the logistics department to load the complete kit and ship it to the construction site.

At the construction site, the kit is unloaded by the logistics team of company X, which checks whether there is any missing items. Next, the production manager schedules the tasks associated with the kits at the weekly planning. The installation of gutters is usually broken down into four operations: transport of the material to the place of use; installation of the support (threaded bar and metallic angle brackets) for the guttering; pre-assembly of the gutters at the place of use, which involves welding of the 3-meter gutters; and connecting the gutters to the respective supports. Upon completion, a final inspection is made by the customer.

\section{USING FRAM FOR ANALYSING THE CHAIN OF WASTES}

Figure 1 presents the FRAM model drawn up to represent this case, for which a total of 17 functions were identified. The functions represented by rectangles, such as $<$ Schedule the Manufacturing of Components>, correspond to the functional boundaries of the system. These boundaries have been arbitrarily defined by the researchers, and only the input or output aspects of the corresponding functions have been described at the model. By contrast, functions represented by hexagons, such as <Manufacture components $>$ have two or more of their aspects described in the model. The aspects are represented by the initials at the corners of the hexagons, as follows: O-output, I-input, R-resource, Pprecondition, T-time, and C-control. 
The waves inside some hexagons indicate that there was variability in the function's output in terms of time, precision, or both. The meaning of the colours of the borders of the hexagons is as follows: green - administrative functions that occur outside the construction site; grey - production functions that occur outside the construction site; blue - production functions that occur in the construction site; purple - administrative functions that occur in the construction site; and red - function carried out by the client. This variety of functions and actors that perform them highlights the rich nature of the interactions in the chain of wastes, which makes it less controllable. The orange lines display the couplings associated with variability propagation across the system, and the numbers inside the hexagons represent the sequence of this propagation. The icons next to the hexagons convey the types of wastes that manifest in each function. It is worth noting that wastes were only visible to the client at the function $<$ Use of the Building $>$, where a waste played out physically as a defective product.

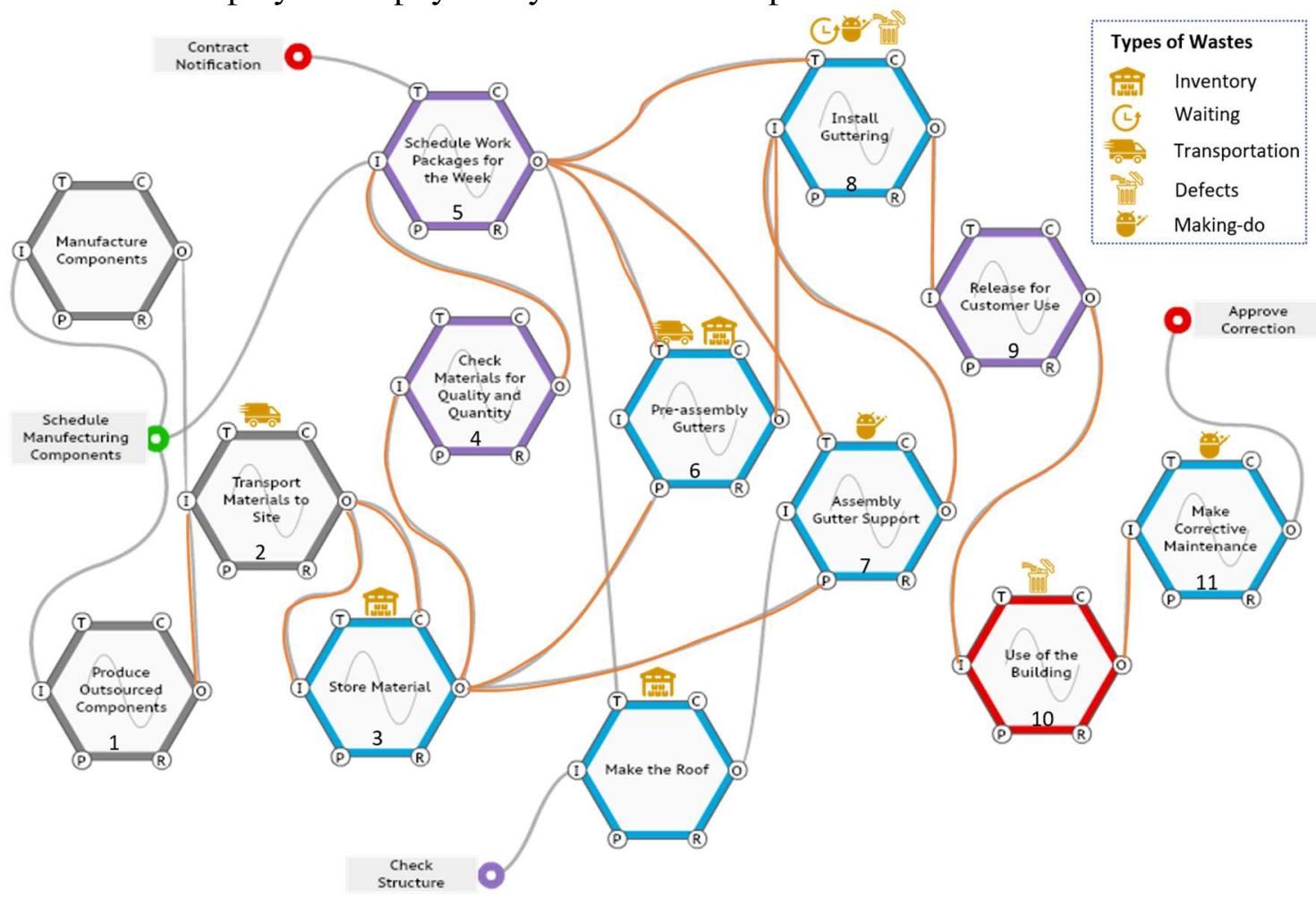

Figure 1- FRAM model for the chain of wastes

According to Figure 1 the chain of wastes started with the output variability, in terms of time, of $<$ Produce Outsourced Components $>$. Indeed, the outsourced company did not produce the components for supporting the guttering within the agreed deadline. This variability was amplified when the construction site's team responsible for $<$ Check Materials for Quality and Quantity> failed to identify that delay, thus hindering the production manager's early awareness of the missing components. As such, that manager wrongly assumed that the required components had been delivered. Thus, through the function $<$ Schedule Work Packages for the Week $>$ he hired workers for the installation of gutters. However, after realizing the problem, and as to avoid workers being idle, the manager opted for starting $<$ Pre-assembly Gutters $>$ even in face of missing supplies. This decision first led to transportation waste. As guttering is a fragile component, it had to be carefully transported to a safe place for storage, thus preventing any other gangs from damaging it when working on the site. 
In fact, this situation could have been more effectively addressed provided company $\mathrm{X}$ had an alternative supplier on standby for the threaded bar and the metallic angle bracket. Experiences in previous projects of company X suggested that the main supplier could not be fully trusted to meet the agreed delivery deadline - the risk of this occurring again had been underestimated. With one week remaining to the expiration of the building delivery deadline and with the imminent notification of the client to apply the contractual fine, the solution devised was to improvise other materials to replace the missing components. Thus, workers improvised the use of metallic braces which are normally used as a strap to package the tile coil (Figure 2). The function <Assemble Gutter Support $>$ accounted for the first manifestation of making-do as it involved the improvisation of the brace, implying in output variability both in terms of time and precision. This resulted in another waste as the team responsible for <Install Guttering $>$ was waiting for the completion of that task. A second manifestation of making-do is represented by the imprecise output of $<$ Install Guttering $>$. Precision was hindered as the gutter was not placed to rest on the position that maintained the required functionality - positioning was improvised to some extent as a result of the previous improvisation in the assembly of the gutter support. After the end of the task, a formal delivery to the customer was carried out, then being followed by $<$ Use of the Building $>$.

However, two months after the completion of the works, a leak in the guttering caused the client to notify company $\mathrm{X}$ to $<$ Make Corrective Maintenance $>$. As the construction solution of the guttering was improvised, a new improvisation (and third manifestation of making-do) had to be conducted so that the updated section of the gutter would be similar to the other sections.
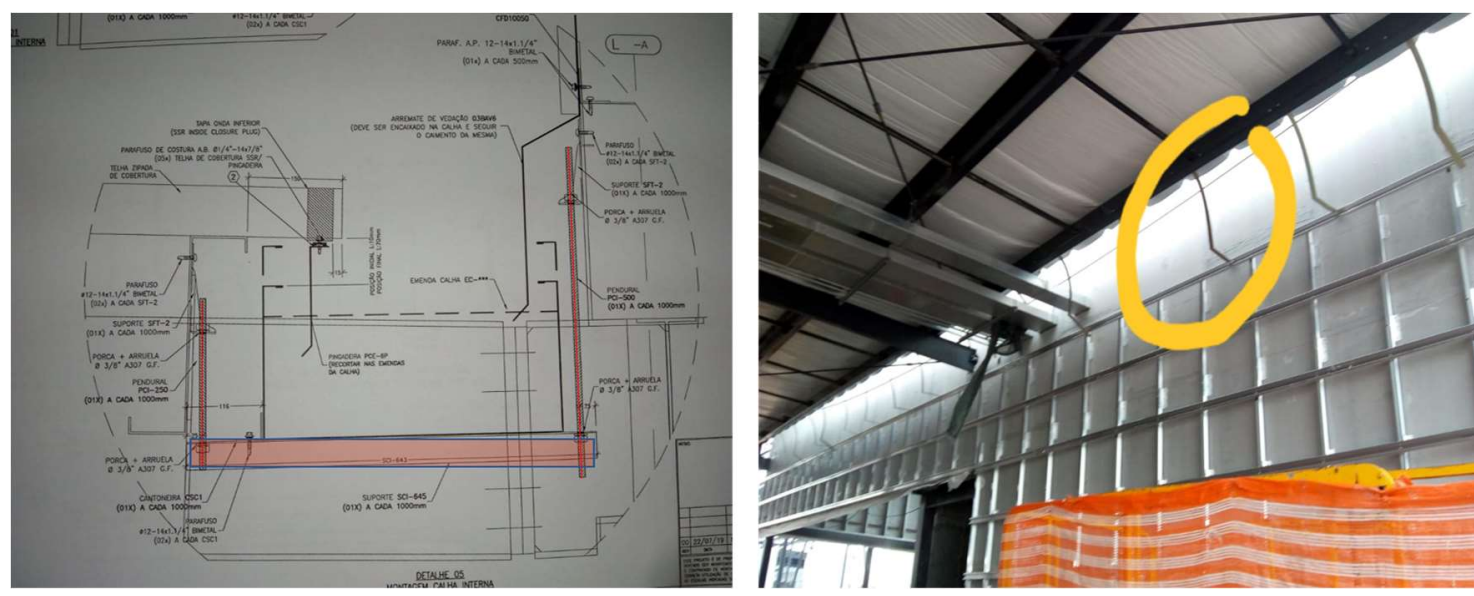

Figure 2: Left:missing items in red. Right: improvised metallic brace is highlighted

The developed model indicates that making-do played out in the later stages of the chain of wastes - i.e. in functions at the 6th, 7th, and 11th steps of the chain (see Figure 1). As such, making-do was not a primary waste, as there were several other wastes and undesired variability sources at earlier stages of the chain. Furthermore, the moderating role played by making-do should be stressed. On the one hand, making-do prevented the application of a contractual fine, thus providing a temporary compensation for upstream variability sources and wastes. On the other hand, making-do added variability downstream in the chain of wastes, triggering even more making-do and other types of wastes. 


\section{DISCUSSION}

\section{THE ROLE OF SLACK FOR COPING WITH MAKING-DO}

Making-do was seen by the construction workers and managers as a last resource to avoid task's delay and the resulting contractual fine. However, a failure in meeting the anticipated preconditions (i.e. make-ready) to start a task must not necessarily imply in making-do. Wastes were amplified as Company $\mathrm{X}$ was not prepared to promptly respond to delays in the delivery of supplies, even though past experience suggested that delays were likely to occur. In this respect, resilient performance would have benefited from the availability of slack, which contributes to coping with variability. Slack is a "cushion of resources" that an organization can use to counter threats as well as to exploit opportunities (Bourgeois, 1981). The concept of slack has a broader scope than the term buffer, as the former is not limited beforehand to any specific type of resource. Unlike buffer, which usually implies in using time, inventory or capacity as a resource to cope with variability (Hamzeh et al., 2012), slack accounts for any type of resource (e.g. perspectives to solve a problem, degrees of freedom in standard operating procedures, multifunctional workers, redundant equipment), ideally intentionally designed for addressing variability (Saurin and Werle, 2017). For example, in the case studied, a number of approaches could have provided slack, such as: (i) alternative suppliers to provide the threaded bar and angle bracket; (ii) stocks of these components either in the supplier's or in the contractor's warehouses; (iii) borrowing the components from another project; and (iv) redundant checks to detect failures in the delivery of supplies on the construction site, such as by using different professionals and types of inspections.

Figure 3 shows the proposed role for slack as a safety margin between make-ready and making-do. Make-ready is a process of identification and removal of constraints that provides the fulfilment of preconditions before starting a task (Pikas et al., 2012). However, in complex systems unexpected variability is rife and therefore a margin is necessary to prevent a failure in making-ready from immediately becoming a waste of making-do.

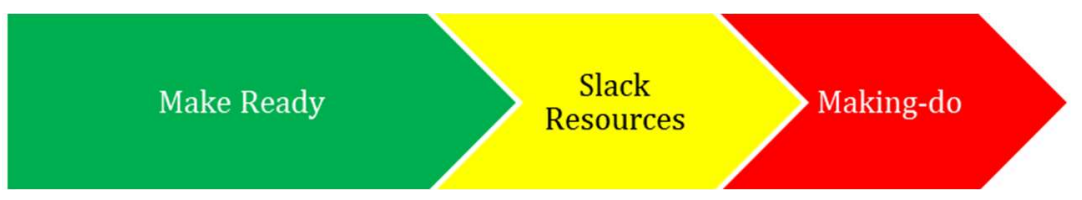

Figure 3: Slack as an approach for preventing a failure in making-ready from becoming a waste of making-do (adapted from Pikas et al. 2012)

In Figure 3, slack appears in yellow as to emphasize that the simple need for deploying it should be interpreted as a warning signal that performance is no longer within the boundaries expected in standardized operating procedures. The effectiveness of using slack will determine whether the system bounces back to the desired state (green) or migrates towards making-do waste (red). It is worth noting that the role played by slack (or buffers) had not been highlighted by earlier studies of making-do in lean construction (Formoso et al., 2011; Fireman et al., 2013; Ibarra et al., 2016). While Koskela (2004) described making-do as a negative buffer, he did not stress the need for real buffers as a countermeasure. In turn, Ebbs and Pasquire (2018) acknowledged the need for slack to cope with failures in making-ready, even though they made no theoretical connection with making-do. Of course, variability should be ideally removed in the first place, eliminating the need for slack - e.g. this could have implied in replacing an unreliable 
supplier before starting the construction project. However, experience from everyday work in complex systems indicates that a substantial level of variability is unavoidable and therefore preparing for that is advisable (Bertelsen and Koskela, 2005).

\section{HOW FRAM PRINCIPLES PLAYED OUT IN THE CASE STUDY}

Analysing the results from the viewpoint of the four FRAM principles, some additional insights can be obtained. The principle of equivalence suggests that the same factors that contributed to wastes could have contributed to successful performance under other circumstances. For example, it can be hypothesized that a more effective solution could have been devised if company $\mathrm{X}$ had records of lessons learned from similar situations. In fact, the point of the principle of equivalence is that improvisations do not necessarily need to imply in making-do - i.e. improvisation has a dual-character. This is reinforced by Hamzeh et al. (2018), which found that personal experience, and organizations that empower employees and allow experimenting, have a positive effect on the outcomes of improvisation.

The principle of approximate adjustments can be illustrated by making-do waste itself, as by definition it does not produce a timely and precise outcome, due to resource constraints. As to the principle of emergence, this can be illustrated by the creation of unanticipated activities - e.g., transportation of the guttering components for a temporary storage location on the construction site. These activities only existed because the output variability of some functions triggered new interactions between production and management functions. In fact, making-do waste incurred when installing gutters without all standard components is an emergent phenomenon as well, as it had new properties (i.e. client dissatisfaction) that did not exist in the upstream functions. Lastly, the principle of functional resonance means that making-do occurred due to the combined variability of several functions under a certain context, rather than being a linear chain of events traceable to a single root cause.

\section{CONCLUSIONS}

FRAM provided a functional and holistic view of the chain of wastes, indicating that it might involve wastes that occur outside of the construction site and continue into the phase of using the building. Also, FRAM showed how the variability of multiple functions, performed by different actors in production and managerial functions, combined for the emergence of making-do and other wastes in the chain of waste.

This study indicated that making-do played a moderating role in the chain of wastes. While it compensated for wastes arising from the initial stages of the chain of wastes (i.e. it prevented a contractual fine from being applied due to delays in the schedule) it created new wastes downstream. Another insight from this research is that the prevention and mitigation of the impacts of making-do might benefit from the anticipation of variability sources and the design of corresponding slack resources.

Two limitations of this study must be mentioned. First, the use of a single case study limited the understanding of the implications of FRAM for understanding making-do in the context of chains of wastes. Second, no quantitative data were gathered to assess the impacts of the wastes nor the costs associated with slack resources. As a consequence of these limitations, future studies could address: (i) cost-benefit analysis of providing slack in construction projects; (ii) the use of FRAM for investigating making-do in a broader set of cases, with various levels of complexity - these studies could further explore the nature of the interactions (Saurin and Patriarca, 2020) between functions in a chain of 
wastes, such as by considering the speed of waste propagation; and (iii) FRAM might be used proactively (instead of retrospectively, as in this study), as a risk assessment approach for the identification of variabilities that can give rise to wastes. This can also support the identification of where and how much slack is necessary in construction projects.

\section{REFERENCES}

Bertelsen, S; Koskela, L. (2005).“Approaches to Managing Complexity in Project Production". In: Proc 13 ${ }^{\text {th }}$ Annual Conference of the International Group for Lean Constructuion. Sydney, Australia.

Bourgeois, J. (1981). "On the measurement of organizational slack". Academy Management Review 6(1): 29-39.

Bølviken, T.; Rooke, J.; Koskela,L. (2014). “The wastes of production in construction- A TFV Based Taxonomy". In: Proc. $22^{\text {nd }}$ Annual Conference of the International Group for Lean Construction, Oslo, Norway.

Bølviken , T.; Koskela, L. (2016)."Why hasn't waste reduction conquered Construction?" In: Proc. 24 $4^{\text {th }}$ Ann. Conf. of the International Group for Lean Construction, Boston, MA, USA.

Clay-Williams, R., Hounsgaard, J., \& Hollnagel, E. (2015). "Where the rubber meets the road: using FRAM to align work-as-imagined with work-as-done when implementing clinical guidelines". Implementation Science, 10(1), 125.

Ebbs, P.J.; Pasquire, C.L. (2018).“Make Ready Planning Using Flow Walks: A New Approach to Collaboratively Identifying Projects Constraints". In:Proc. $26^{\text {th }}$ Annual Conference of International Group for Lean Construction. Chennai, India.

Fireman, M.C.T.; Formoso, C.T.; Isatto, E.L. (2013)."Integrating production and quality control: monitoring making-do and informal work packages". In: Proc. $21^{\text {th }}$ Annual Conference of the International Group for Lean Construction, Fortaleza, Brasil.

Formoso, C.T.; Sommer, L.; Koskela, L.; Isatto, E.L. (2011).“An exploratory study on the measurement and analysis of making-do in construction sites". In: Proc. $19^{\text {th }}$ Annual Conference of the International Group for Lean Construction, Lima, Peru.

Formoso, C.T.; Bølviken, T.; Rooke, J.; Koskela, L. (2015).“A Conceptual Framework for the Prescriptive Causal Analysis of Construction Waste". In: Proc. $23^{\text {th }}$ Ann. Conf. of the Int'l. Group for Lean Construction, Perth, Australia.

Ibarra, J.V.; Formoso, C.T.; Lima, C.;Mourão, A.; Saggin, A. (2016)."Model for integrated Production and Quality Control: Implementation and Testing Using Commercial Software Applications". In: Proc 24 $4^{\text {th }}$ Annual Conference of International Group for Lean Construction. Boston, USA.

Hamzeh, F.; Ballard, G.; Tommelein, I, D. (2012). "Rethinking Lookahead Planning to Optimize Construction Workflow". Lean Construction Journal, pp15-34.

Hamzeh, F.R.; Faek, F.; AlHussein, H. (2018). "Understanding improvisation in construction through antecedents, behaviours and consequences". Construction Management and Economics, Doi: 10.1080/01446193.2018.1497189.

Hollnagel, E. (2012). "FRAM: the Functional Resonance Analysis Method: Modeling complex socio-technical systems". Farnham: Ashgate.

Hollnagel, E. (2013). "An application of the Functional Resonance Analysis Method (FRAM) to risk assessment of organisational change". Swedish Radiation Safety Authority, Stockhol. 
Koskela, L. (2004). "Making-do - The Eighth Category of Waste". In: Proc. $12^{\text {th }}$ Annual Conference of the International Group for Lean Construction, Denmark.

Koskela, L.; Bølviken, T.; Rooke. (2013). "Which are the wastes of construction?". In:Proc. $21^{\text {th }}$ Annual Conference of the International Group for Lean Construction, Fortaleza, Brazil.

Ohno, T. (1988).“Toyota Production System: Beyond Large Scale Production". Productivity Press, Cambridge, MA.

Pikas, E. , Sacks, R. \& Priven, V. (2012). "Go or No-Go Decisions at the Construction Workface: Uncertainty, Perceptions of Readiness, Making Ready and Making-Do". In:Proc. $20^{\text {th }}$ Annual Conference of the International Group for Lean Construction. San Diego, USA, 18-20 Jul 2012.

Saurin, T.A. (2016). "The Fram as a Tool for Modelling Variability Propagation in Lean Construction". In: Proc. 24 $4^{\text {th }}$ Ann. Conf. of the International Group for Lean Construction, Boston, MA, USA.

Saurin, T.A; Werle, N.B. (2017). "A framework for the analysis of slack in sociotechnical systems". Reliability Engineering and Systems Safety, 167, 439-451

Saurin, T. A.; Patriarca, R. 2020. "A taxonomy of interactions in socio-technical systems: A functional perspective”. Applied Ergonomics, 82, 102980. 